\title{
REVISÃO DA LITERATURA: AVANÇOS E PERSPECTIVAS NO TRATAMENTO DE ALZHEIMER EM IDOSOS COM ÊNFASE NA BARREIRA HEMATOENCEFÁLICA
}

\author{
Alexander Carcelen Martins ${ }^{1}$ \\ Giovanna Menegatti Teixeira ${ }^{2}$ \\ Mariana Yukie Oshiro ${ }^{3}$ \\ Beatriz Silva Santos ${ }^{4}$
}

RESUMO: Objetivo: A barreira hematoencefálica (BHE) é um protetor natural do sistema nervoso central (SNC), mas também pode ser um fator limitante para fármacos devido a sua alta seletividade. Uma maior permeabilidade e menor seletividade da BHE não mostrou ser a resposta para melhorar os tratamentos medicamentosos de pacientes com doença de Alzheimer (DA) e outras demências, pois dados encontrados apontam que uma maior permeabilidade pode ser fator contribuinte para o avanço de demências. $O$ presente trabalho busca trazer os últimos avanços no tratamento da DA e fomentar a discussão sobre tais tratamentos e avanços, abordando especificamente a BHE. Abordaremos os aspectos fisiológicos da $\mathrm{BHE}$ relacionando-os com os fármacos usados para o tratamento, abordando também as características dos fármacos que dificultam atravessar a BHE. Buscou-se realizar uma revisão principalmente da literatura estrangeira, trazendo os últimos avanços para o tratamento da DA, visto que existe uma quantidade maior de material sobre a BHE em material em inglês do que em português. Métodos: Foi realizada uma revisão da literatura utilizando os seguintes bancos de dados: PubMed, Scielo, Google acadêmico. O período de abrangência usado nas pesquisas foi entre $2005 \mathrm{e}$ 2020. Buscamos revisar também ensaios clínicos atualmente registrados, que buscam estudar a BHE e a DA, na base de dados norte-americana clinicaltrials.gov. Revisamos também capítulos que abordavam a BHE de três livros, sendo um livro de fisiologia e dois livros de farmacologia. Conclusão: Conclui-se que a BHE não pode ficar de fora de pesquisas para DA e outras demências devido a sua extrema importância. Observou-se que a permeabilidade da BHE pode tanto beneficiar os pacientes futuramente se for mensurada e controlada, como pode também prejudicar e colaborar com o avanço das demências se não for totalmente compreendida. A perspectiva para o tratamento da DA sob este prisma da BHE mostrou-se positiva, devido a um aumento encontrado de novas abordagens para tratar, prevenir e compreender a DA.

Palavra-chave: Alzheimer's Disease. Blood-Brain Barrier. Dementia. Ultrasound.

\footnotetext{
IGraduando em farmácia da Universidade Anhembi Morumbi. E-mail: alexcarcelen@hotmail.com

${ }^{2}$ Graduanda em farmácia da Universidade Anhembi Morumbi

${ }^{3}$ Graduanda em farmácia da Universidade Anhembi Morumbi

${ }^{4}$ Graduando em biomedicina da FMU - Faculdades Metropolitanas Unidas
} 
Descritores em português: Doença de Alzheimer. Barreira Hematoencefálica. Demência. Ultrassonografia

Descritores Booleanos: AND

ABSTRACT: Goals: The blood-brain barrier (BBB) is a natural protector of the central nervous system (CNS), but it can also be a limiting factor for drugs due to its high selectivity. Greater permeability and lesser selectivity of the BBB did not prove to be the answer to improve drug treatments for patients with Alzheimer's disease (AD) and other dementias, as data found indicate that greater permeability may be a contributing factor to the advancement of dementias. The present work aims to bring the latest advances in AD treatment and encourage discussion of such treatments and advances, specifically addressing the $\mathrm{BBB}$. We will address the physiological aspects of the $\mathrm{BBB}$, relating them to the drugs used for treatment, also addressing the characteristics of drugs that make it difficult to cross the BBB. We sought to carry out a review mainly of foreign literature, bringing the latest advances for the treatment of $\mathrm{AD}$, as there is a greater amount of material about the BBB in material in English than in Portuguese. Methods: A literature review was performed using the following databases: PubMed, Scielo, Google academic. The coverage period used in the surveys was between 2005 and 2020. We sought to review currently registered clinical trials, which seek to study $\mathrm{BBB}$ and $\mathrm{AD}$, in the North American database clinicaltrials.gov. We also reviewed chapters addressing the $\mathrm{BBB}$ of three books, one book on physiology and two books on pharmacology. Conclusion: It is concluded that the $\mathrm{BBB}$ cannot be left out of research for $\mathrm{AD}$ and other dementias due to its extreme importance. It was observed that the permeability of the BBB can both benefit patients in the future if measured and controlled, and it can also harm and collaborate with the advancement of dementias if it is not fully understood. The perspective for the treatment of $\mathrm{AD}$ from this perspective of the $\mathrm{BBB}$ proved to be positive, due to an increase found in new approaches to treat, prevent and understand AD.

Keywords: Alzheimer's Disease. Blood-Brain Barrier. Dementia. Ultrasound Boolean AND

INTRODUÇÃO:

A doença de Alzheimer (DA) é uma doença neurodegenerativa, acometendo milhões de pessoas ao redor do mundo atualmente, sobretudo idosos. Estimativamente a DA terá acometido I3I milhões de pessoas globalmente no ano de 2050 (CUMMINGS et al., 2020). Tendo seu início lento e progressivo, os primeiros sintomas da DA são, frequentemente, confundidos com dificuldades corriqueiras do nosso cotidiano, desencadeadas pela idade 
Revista Ibero- Americana de Humanidades, Ciências e Educação- REASE

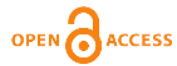

até mesmo em pessoas saudáveis, como esquecer-se de objetos e tarefas, o que torna uma doença de difícil diagnóstico e prevenção. A perda de volume de tecido cerebral, as placas senis, que são deposições extracelulares do peptídeo beta-amilóide, emaranhados neurofibrilares, compostos pela proteína tau hiperfosforilada, diminuição da neuroplasticidade e neuroinflamação, com posterior alteração na demanda de neurotransmissores, são exemplos

de biomarcadores desta doença (PAULA et al., 2009). Uma vez identificados os sintomas e tendo o diagnóstico, recomenda-se o uso majoritariamente de anticolinesterásicos (SHARMA, 2019).

A barreira hematoencefálica (BHE) é um dos fatores limitantes principais na distribuição de medicamentos no sistema nervoso central (SNC) (PARDRIDGE, 20I2). Esta barreira é como um sistema de proteção complexo e seletivo do próprio SNC (MENDONÇA et al., 2015). Ao se deparar com esta barreira, as moléculas, cujo objetivo é atingir um local específico no encéfalo, encontram dificuldade em atravessá-la, limitação esta que muitos medicamentos cujo alvo é o SNC têm em comum, o que inclui os anticolinesterásicos. Os medicamentos para a DA atualmente em uso são mencionados em diversos estudos como tendo eficácia limitada justamente pela dificuldade em cruzar a BHE. Este artigo tem o objetivo de analisar a capacidade e necessidade de fármacos em atravessarem a BHE. Foram encontrados dados mostrando êxito no aumento da concentração de moléculas no SNC após ser possível abrir a BHE temporariamente (MENDONÇA et al., 2015; RAYMOND et al., 2008), mostrando que este é um campo com potencial a ser explorado no tratamento da DA. Encontrou-se também estudos abordando

especificamente a $\mathrm{BHE}$, buscando compreende-la mais afundo, fomentando este outro aspecto do SNC para novos possíveis tratamentos no futuro.

Ao abordar as diferentes terapias medicamentosas e não-medicamentosas para a DA, o presente trabalho discutirá os achados em estudos sobre possíveis as limitações conhecidas dos fármacos tão bem quanto a eficácia de tais tratamentos, e relacioná-las com as características físicas microscópicas da BHE que contribuem com tais limitações. 


\section{Métodos}

\section{I.I. Bases de dados}

Realizamos uma revisão literária utilizando os seguintes bancos de dados: PubMed (National Library of Medicine), Scielo (Scientific Eletronic Library Online) e Google acadêmico. De acordo com os critérios de inclusão, foram revisados I8 artigos nas bases de dados.

\subsection{Ensaios clínicos}

I.3. Realizamos 2 pesquisas no clinicaltrials.gov (NIH - U.S. National Library of Medicine)

Pesquisa I: foram utilizados os seguintes filtros: Other terms: Blood-Brain Barrier. Fase do estudo: estudo de fase inicial I (antiga fase o), estudo fase I, estudo fase 2, estudo fase 3, estudo fase 4 e N/A (Not Applicable). Filtros de recrutamento utilizados: recrutando participantes, ainda não recrutando participantes, ativo mas não recrutando, recrutamento concluído, inscrição mediante convite, e suspenso. Participantes de ambos os sexos, sem restrição de idade, considerando também pacientes saudáveis nos testes. Data de início igual ou superior a or/or/2015. Esta pesquisa considerou todos os países com testes clínicos registrados no clinicaltrials.gov. Dentre os 15 ensaios clínicos que foram encontrados com os filtros acima, foram incluídos somente cinco neste artigo por critérios de inclusão, sendo que o critério utilizado foi incluir nesta revisão apenas os ensaios clínicos que abordem a BHE mais diretamente.

Pesquisa 2: filtro utilizado: Disease or Condition: Alzheimer e Other terms: Ultrasound.

Utilizou-se esta base de dados Norte-Americana, pois o Estados Unidos é o país com o maior número de testes clínicos em andamento registrados.

\section{I.4. Livros}

Revisamos capítulos que abordavam a BHE de três livros, sendo um livro de fisiologia e dois livros de farmacologia 
Revista Ibero- Americana de Humanidades, Ciências e Educação- REASE

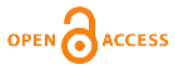

\section{Acetilcolina e Acetilcolinesterase}

Para manter a integridade e também um bom funcionamento dos neurônios, são necessárias proteínas e neurotransmissores. Um dos neurotransmissores de grande interesse é a acetilcolina (ACh); um mediador químico de sinapses no SNC e sistema nervoso periférico (SNP) (VENTURA et al., 20ıo).

Mediadores químicos podem ser responsáveis pela neurotransmissão colinérgica. Sendo liberados nas fendas sinápticas, podem desencadear respostas tanto inibitórias quanto excitatórias ao se ligarem nos receptores colinérgicos muscarínicos ou nicotínicos. Ao ser acometido com a doença de Alzheimer, estas proteínas e neurotransmissores podem sofrer alterações, gerando efeitos excito-tóxicos ou sofrendo déficits, e também sofrendo alterações estruturais, levando o neurônio a morte e posterior declínio cognitivo (CLARK et al., 2013).

A enzima acetilcolinesterase (AChE), localizada na fenda sináptica, quebra a ACh em acetato e colina. No sistema nervoso central e periférico, são encontrados ambos receptores colinérgicos, que usam a acetilcolina como neurotransmissor. A perda de

neurônios colinérgicos é uma das características principais relacionadas à perda de memória na doença de Alzheimer. Ao inibir a acetilcolinesterase no SNC, há uma melhora na transmissão colinérgica que é o mecanismo de ação dos medicamentos amplamente usados na DA, que são inibidores da acetilcolinesterase, são exemplos: galantamina, donepezila e rivastigmina, buscando promover um uso prolongado do neurotransmissor, desencadeando assim uma resposta em ambos receptores (DE FALCO et al., 2015; CLARK et al., 2013).

\section{Barreira Hematoencefálica (BHE)}

A BHE é um protetor do próprio SNC e também um fator limitante natural de moléculas, com permeabilidade altamente seletiva de substâncias que podem vir a ser tóxicas/nocivas, inclusive moléculas de medicamentos e microrganismos (KATZUNG e TREVOR, 2017; CARTER et al., 2020).

No corpo humano, as trocas e transporte de nutrientes ocorrem pela circulação sanguínea, que é composta por vasos sanguíneos; artérias, veias e capilares sanguíneos. Os capilares são os vasos de menor calibre, ocupando os locais mais escusos do corpo humano. 
Revista Ibero- Americana de Humanidades, Ciências e Educação- REASE

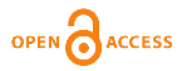

A BHE, que é formada por células endoteliais nos capilares formando junções íntimas (JI), por astrócitos e também por células chamadas pericitos, é a responsável pela troca entre a circulação sistêmica e o SNC. Esta composição da BHE é responsável pela ausência de passagens entre as células, gerando esta alta seletividade da BHE (CALAPAI et al., 2020).

A dopamina é um bom exemplo de fármaco que não pode atravessar a $\mathrm{BHE}$ no tratamento de Parkinson. Uma forma de se ter êxito no transporte de dopamina através da BHE é utilizando o precursor dopaminérgico L-dopa, que posteriormente é metabolizado em dopamina. Outro exemplo são os anti-histamínicos mais apolares, os de primeira geração, que podem causar sonolência. Esta característica deve-se ao fato de que moléculas lipossolúveis possuem maior capacidade em atravessar a BHE do que as moléculas mais polares dos anti-histamínicos de segunda geração que são hidrossolúveis (KATZUNG e TREVOR, 2017; SILVERTHORN, 2017).

Um outro exemplo de pró-fármaco encontrado na literatura é a droga Gln-ıo62, um pró-fármaco da Galantamina, mais lipofílico, e que, da mesma forma que a L-dopa, é ativado posteriormente ao adentrar no encéfalo mais facilmente (BAAKMAN et al., 2016).

Este aumento da lipofilicidade é uma estratégia de transformar moléculas hidrofílicas em lipofílicas, adicionando grupos lipídicos ou grupos funcionais na molécula do fármaco. Conferindo assim capacidade à molécula de adentrar através de transporte passivo na BHE (SCARPA e BELLEATO, 2018).

Um melhor entendimento do funcionamento da BHE é necessário para o desenvolvimento de novas drogas que buscam atravessá-la. Algumas funções essenciais da BHE são: protegê-la da entrada de agentes potencialmente nocivos, permitir a entrada do necessário, e secretar substâncias. Relacionado ao transporte de drogas através da $\mathrm{BHE}$, existe a difusão transmembrana (mecanismo não-saturável) no qual a lipossolubilidade e baixo peso molecular são características que favorecem o transporte através da BHE (BANKS, 2009).

Em um estudo que demonstrou o papel dos oligômeros beta-amilóides, após serem injetados no encéfalo de macacos e camundongos, os pesquisadores observaram como os oligômeros se acumularam em regiões associadas com a memória, comparando posteriormente os resultados com a DA em humanos. Observando, assim, que existe ainda 
Revista Ibero- Americana de Humanidades, Ciências e Educação- REASE

a necessidade adicional da criação de modelos animais com DA, que mais se aproximem da DA em humanos e não somente a necessidade de entender a BHE para o desenvolvimento de novos medicamentos ou tratamentos (GERMANO et al., 2020).

\section{Permeabilidade da BHE e Demências}

\section{I.I. Traumatismo craniano}

Traumatismos podem ser um fator de risco para o desenvolvimento de doenças neurodegenerativas a longo prazo em pacientes sobreviventes de traumas deste tipo, devido a deterioração da BHE iniciada pelo próprio traumatismo (ROSA et al., 2020). Diversas doenças neurodegenerativas podem ser devido a problemas nas disfunções da BHE, como o Alzheimer e Parkinson, por exemplo (GAWDI e EMMADY, 2020).

\subsection{2. Óxido de grafeno reduzido e a BHE}

Pesquisadores utilizaram a forma reduzida do óxido de grafeno, obtida em laboratório buscando analisar a integridade da BHE, administrando o corante azul T-I482 por infusão intravenosa em camundongos já com administração de rGO (óxido de grafeno reduzido) e camundongos sem administração de rGO. O corante T-I482 é um corante naturalmente incapaz de atravessar a BHE. Observaram o vazamento do corante somente nos encéfalos com rGO após 15 minutos, I hora e 3 horas da administração, constatando abertura temporária da BHE (MENDONÇA et al., 2015).

Sobre as características gerais relatadas sobre o comportamento da rGO in vivo, puderam afirmar que é um composto que adentra progressivamente no encéfalo e que todas as alterações observadas retornavam ao estado inicial após 7 dias da administração do mesmo. Apesar de fornecerem dados a favor deste método reconhecem que ainda existem pontos a serem investigados, como a toxicidade do rGO (MENDONÇA et al., 2015).

\subsubsection{Ultrassom com administração de Microbolhas (FUS-MB; Focused Ultrasound with Microbubbles) e a BHE}

Em outro teste utilizando modelos de camundongos, utilizaram a energia das ondas sonoras do ultrassom para indicar o aumento da entrega de um corante em certas regiões dos hemisférios dos encéfalos dos camundongos em estudo pós-morte, mostrando, assim, o 
Revista Ibero- Americana de Humanidades, Ciências e Educação- REASE

extravasamento do corante através da BHE. Este estudo utilizou a ressonância magnética para auxiliar no monitoramento da ruptura da BHE, e as microbolhas administradas expandem e contraem auxiliando na abertura da BHE. Foi usado o corante azul de Tripano, que foi mencionado ser um corante capaz de se ligar as amiloides, e da mesma forma que do estudo anterior, foi citado como incapaz de atravessar a BHE naturalmente devido as suas características físicas (RAYMOND et al., 2008; JAMES et al., 20II).

\subsubsection{Agentes quelantes e a BHE}

Alguns metais como $\mathrm{Zn}, \mathrm{Cu}$ e Fe por exemplo, possuem a capaciade de complexarem com as placas Beta milóides no encéfalo de pacienes com DA, e gerar radicais livres, que além de contribuir com estresse oxidativo pode dificultar a remoção das placas amillóides. Desta forma, moléculas quelantes (possuem a capacidade de capturar iôes metálicos e tratar intoxicações por metais), também são testadas para tratamento da DA, porém muitas substâncias quelantes não possuem a capacidade de atravessar a BHE. Como a desferroxiamina, usada no tratamento de hemocromatose, que potencialmente auxiliaria na remoção de metais do encéfalo de paciente com DA, possui elevado peso molecular e característica hidrofílica, e não lipofílica o que é desejado para cruzar a BHE. Outro quelante encontrado na literatura é o clioquinol, cujas características são: lipofilicidade, tamanho pequeno e capaz de cruzar a BHE. A deferiprona, outro agente quelante testado para auxiliar

no tratamento da DA, foi descrito como uma molécula pequena e lipofílica (DE FALCO et al., 2016).

\section{Peso molecular de fármacos e substâncias e a importância para a BHE}

Um baixo peso molecular para moléculas lipofílicas atravessarem a BHE está entre os tamanhos 400 - 6oo Daltons (SCARPA E BELLEATO, 2018), ou menores de 500 Daltons de tamanho (GAWDI, 2020).

$\mathrm{Na}$ tabela abaixo veremos as substâncias citadas que são capazes, ou não, de atravessar a BHE e suas características.

Tabela I. Massa molecular de substâncias químicas e fármacos para Alzheimer e outras demências 
Revista Ibero- Americana de Humanidades, Ciências e Educação- REASE

\begin{tabular}{llll}
\hline Substância & Massa molecular & Lipofílico & Atravessa a BHE \\
\hline Desferroxiamina & $560,684 \mathrm{~g} / \mathrm{mol}$ & Não & Não \\
\hline Deferiprona & $139,152 \mathrm{~g} / \mathrm{mol}$ & Sim & Sim \\
\hline Clioquinol & $305,5 \mathrm{~g} / \mathrm{mol}$ & Sim & Sim \\
\hline Corante azul de tripano & $872,88 \mathrm{~g} / \mathrm{mol}$ & $*$ & Não \\
\hline L-Dopa (Levodopa) & $197.18 \mathrm{~g} / \mathrm{mol}$ & Sim & Sim \\
\hline Galantamina & $287.353 \mathrm{~g} / \mathrm{mol}$ & $*$ & Sim \\
\hline Rivastigmina & $250.336 \mathrm{~g} / \mathrm{mol}$ & $*$ & Sim \\
\hline Donepezila & 379.492 & Sim
\end{tabular}

(*): informação desconhecida

Fonte: (WISHART et al., 2017)

\section{4-Medicamentos para DA atualmente em fase de testes}

Existe um número consideravelmente alto de medicamentos explorando outras formas de tratamento registrados no clinicaltrials.gov, indo além dos meios mais conhecidos e utilizados para tratar a DA. Muitos dos agentes candidatáveis em fase de testes são anticorpos monoclonais, como por exemplo o mais novo medicamento biológico aprovado para DA, o Aducanumab, um anticorpo monoclonal que visa remover as placas beta-amilóides. Outros fármacos buscam a proteção neuronal, alguns agem em fatores vasculares, intervenções epigenéticas, tratam inflamações, neurogênese, entre outros. Observou-se também alguns medicamentos já existentes no mercado, inicialmente usados para tratar outras doenças, tendo seu uso original modificado ou utilizado para auxiliar no tratamento da DA, por exemplo os medicamentos Mirtazapina, Valaciclovir, Metilfenidato, Tacrolimo e Metformina (CUMMINGS et al., 2020). Como a DA não é 
Revista Ibero- Americana de Humanidades, Ciências e Educação- REASE

totalmente compreendida, principalmente o que causa a doença, estas novas abordagens são de extrema importância.

\section{I. Ensaios clínicos registrados no clinicaltrials.gov (NIH - U.S. National Library of Medicine)}

\subsubsection{Pesquisa I}

Ao utilizar o termo Blood-Brain Barrier (termo em inglês para Barreira hematoencefálica), buscamos descobrir se existem testes clínicos mais específicos que abordem a DA juntamente com a BHE, visto que muitos estudos mostram uma relação estreita entre a integridade da BHE com declínios cognitivos e a capacidade de fármacos adentrarem ou não no encéfalo através dela. Veremos a seguir os ensaios clínicos encontrados.

- Inflamação no encéfalo

Este ensaio clínico acredita poder atenuar os processos inflamatórios gerados pela ativação das micróglias, que são células imunológicas do encéfalo com importante função justamente em processos inflamatórios, através do consumo de óleo do bagaço de oliva, reduzindo, assim, o risco de desenvolvimento e progressão da DA. De acordo com a pesquisa, o óleo do bagaço de oliva é capaz de regular o estresse oxidativo e as respostas inflamatórias advindas das micróglias, protegendo o encéfalo. Uma característica comum da DA, o acúmulo de proteína beta-amilóide, pode estar relacionado a alterações na $\mathrm{BHE}$ que podem ocorrer após neuroinflamação (PERONA; clinicaltrials.gov (NIH), 202I).

As inflamações voltam a ser exploradas em outro estudo na França. Neste estudo buscaram compreender melhor a relação entre a inflamação central e periférica em pacientes depressivos com comportamentos suicidas, e citam que estudos pós-morte evidenciaram rastros de um aumento da ação das micróglias no tecido cerebral em pacientes suicidas, e como visto no estudo anterior a este, as micróglias podem ser responsáveis por ações inflamatórias. A BHE é abordada neste estudo como tendo papel importante agindo como um filtro capaz de garantir o adequado funcionamento das funções neuronais e que inflamações podem alterar a BHE (COURTET; clinicaltrials.gov (NIH), 2021).

\section{- Doença Renal Crônica (DRC)}


Revista Ibero- Americana de Humanidades, Ciências e Educação- REASE

Pacientes com DRC em estágio final podem apresentar funções cognitivas prejudicadas juntamente à DRC. Conforme as funções renais se deterioram, toxinas podem se acumular, intoxicando o encéfalo do paciente causando dano à parte cognitiva. $\mathrm{O}$ principal objetivo desse estudo clínico será avaliar a permeabilidade da BHE na DRC em fase final. A toxina Indoxyl Sulfate (IS) será a toxina analisada no ensaio (GARRIDOPRADALIE; clinicaltrials.gov (NIH), 2020).

\section{- Anemia}

A permeabilidade da BHE também é alvo de um estudo em fase inicial, em pacientes com anemia moderada a grave e outro grupo controle, nos Estados Unidos, em um ensaio clínico. Entre os objetivos deste estudo clínico, estão: analisar o fluxo de sangue normal no encéfalo, volume de matéria branca, oxigênio e conectividade funcional, mais precisamente

como são afetados pela anemia. Os participantes irão passar por ressonância magnética (RM) e testes cognitivos após serem submetidos a reposição de carboximaltose férrica (WOOD; clinicaltrials.gov (NIH), 2020).

\section{- Intervenções multimodais}

Uma intervenção multimodal no estilo de vida com Epigalocatequina-galato (EGCG), um antioxidante natural; um flavonóide, estudará os resultados centrados no paciente. As intervenções multimodais citadas são: nutricional, atividades físicas e cognitivas. De acordo com o que já foi publicado sobre o estudo, as abordagens terapêuticas também buscarão uma melhora da plasticidade sináptica e conectividade funcional nos estágios iniciais da DA. Com este método combinatório, os pesquisadores acreditam ser possível desacelerar o declínio cognitivo com uma eficiência maior do que quando comparado com métodos que utilizam intervenção única no estilo de vida, por exemplo alterar apenas a dieta nutricional ou apenas adicionar exercícios físicos mas não em combinação um com o outro (MOLINUEVO e TORRE, clinicaltrials.gov (NIH), 2020).

\subsubsection{Pesquisa 2}

\section{- Ultrassonografia}


Revista Ibero- Americana de Humanidades, Ciências e Educação- REASE

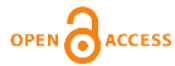

A ultrassonografia é um método antigo, inicialmente usado para outros fins, mas nas últimas décadas, com o avanço da tecnologia e avanço de pesquisas, somados com esta urgência crescente que vemos hoje em encontrar uma forma de combater e compreender melhor a DA, nota-se um interesse crescente no uso da ultrassonografia em aplicação transcraniana. Este teste clínico com ultrassonografia visa testar a segurança e viabilidade deste método tão bem quanto testar se este método altera os níveis da proteína amiloide e os efeitos nas funções cognitivas. Este método para abrir a BHE pode ser útil não somente para aumentar a entrega de medicamentos em locais específicos no encéfalo, como também pode servir para aumentar a segurança do encéfalo realizando o oposto, evitando a ruptura da BHE. Vale ressaltar que por ser um método não invasivo, pode ter a vantagem de trazer comodidade para os pacientes (KONOFAGOU, clinicaltrials.gov (NIH), 2020).

\section{DISCUSSÃO e CONCLUSÃO}

O aumento da expectativa de vida trouxe consigo um aumento nos casos de Alzheimer e outras demências, fazendo com que mais estudos surgissem buscando atender esta urgência, em busca de novas abordagens para tratar, prevenir, retardar o avanço das demências, compreendê-las melhor e fornecer melhora na qualidade de vida dos pacientes.

Ao rodar pesquisas utilizando-se os filtros mencionados nesta revisão, encontramos estudos explorando a importância da BHE também em outras doenças além das demências, com isso conseguimos relacionar características das demências com características destas outras enfermidades, enxergando novas possibilidades de tratamentos futuramente. Pois novas abordagens, por exemplo sobre as diferentes causas de inflamação no encéfalo, podem trazer novas formas de enxergar as inflamações nas demências em si.

A importância sobre novas abordagens e pontos de vistas na demência é observada mais claramente no fato de que, quando se trata de Alzheimer e outras demências, trata-se majoritariamente de idosos, e quando o assunto é a saúde do idoso, pode-se afirmar que dificilmente é lidado somente com uma única enfermidade; o idoso com demência dificilmente sofre apenas com a demência. Nos diferentes estudos clínicos abordados usando o filtro Blood-Brain Barrier, buscamos ressaltar este ponto. $O$ idoso com Alzheimer e outras demências pode sofrer, também, de depressão, que foi citado como outra causa provável de inflamação no encéfalo, que por sua vez pode alterar a 
Revista Ibero- Americana de Humanidades, Ciências e Educação- REASE

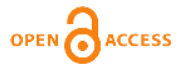

permeabilidade da BHE. O idoso com DA e outras demências pode, da mesma forma, sofrer de DRC, que foi apontado em um estudo clínico como fator para acúmulo de toxinas no corpo que podem gerar declínio cognitivo e danificar a BHE.

Os estudos clínicos com um objetivo mais centralizado, como os que visam unicamente medir a permeabilidade da BHE, também podem servir de apoio para futuras pesquisas de medicamentos para tratar doenças do SNC. Os achados na literatura relacionando ou mencionando única, direta ou indiretamente a $\mathrm{BHE}$ contribuem para o entendimento das perspectivas dos estudos atuais sobre demências, mostrando que existe um avanço no tratamento medicamentoso e no desenvolvimento de métodos que possam auxiliar no diagnóstico recente, o que inclui métodos não invasivos que pode colaborar com a comodidade dos pacientes. Muitos estudos apontam a permeabilidade da BHE como um fator limitante em comum de medicamentos tão bem quanto fator iniciante de desenvolvimento e progressão de danos cognitivos. Nota-se que a permeabilidade citada pode tanto beneficiar se for controlada, quanto prejudicar se não for compreendida e mensurada, permeabilidade esta que pode ser consequência inclusive outras doenças ou traumatismos cranianos.

Podemos concluir também que de um ponto de vista mais molecular, os medicamentos para Alzheimer atualmente no mercado, citados nesta revisão, se encaixam dentro do peso molecular ideal para atravessar a BHE, porém observou-se que mesmo estes medicamentos cujo alvo biológico é o SNC e mesmo tendo um peso molecular adequado, encontram dificuldade para atravessar a BHE.

Métodos preventivos são frequentemente incentivados e fortemente recomendados em outras doenças como sendo a melhor forma de combate à doença, como na diabetes por exemplo. Relacionado à isso, observou-se o interessante fato de que alguns métodos em desenvolvimento e testes clínicos em andamento, poderão vir a servir para monitorar o início do aumento da permeabilidade da $\mathrm{BHE}$, advindo tanto de demências, de outras comorbidades ou de traumatismos, ou mesmo do envelhecimento, que podem contribuir para o desenvolvimento de declínios cognitivos.

Considerando todos aspectos da doença de Alzheimer, esta revisão mostrou que as pesquisas atuais sobre a DA acompanham seu grau de urgência. Foi possível observar um crescente interesse em explorar os diferentes aspectos da doença, como novas substâncias 
Revista Ibero- Americana de Humanidades, Ciências e Educação- REASE

visando tratar de diferentes formas as inflamações por exemplo, ou sob um prisma mais macro novas formas de entender como a BHE funciona.

\section{REFERÊNCIAS}

BAAKMAN, Anne; HARTH, Ellen't; KAY, Denis; STEVENS, Jasper; KLAASSEN, Erica; MAELICKE, Alfred; GROENEVELD, Geert. First in human study with a prodrug of galantamine: Improved benefit-risk ratio?. Journal Alzheimer's \& Dementia: Translational Research \& Clinical Interventions, 20r6. Disponível em: 〈https://doi.org/I0.1016/j.trci.2015.12.003〉. Acesso em: 20 de agosto, 2021.

BELlEATO, Cinzia; SCARPA, Maurizio. Possible strategies to cross the blood-brain barrier. Italian Journal of Pediatrics. 2018 Nov 16 . Disponível em: 〈https://doi.org/10.186/sizo52-018-0563-o〉 Acesso em: 20 de Junho, 2021

BANKS, William. Characteristics of compounds that cross the blood-brain barrier. BMC Neurology, volume 9, Número do artigo: $S_{3}, \quad$ 2009. Disponível

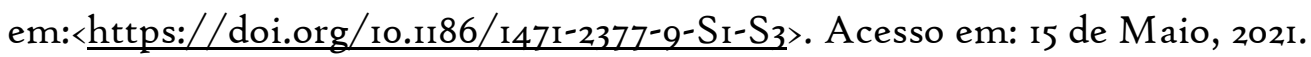

CALAPAI, Fabrizio; CARDIA, Luigi; SORBARA, Emanuela; NAVARRA, Michele; GANGEMI, Sebastiano; CALAPAI, Gioacchino; MANNUCCI, Carmen. Cannabinoids,

Blood-Brain Barrier, and Brain Disposition. Pharmaceutics, 2020. Disponível em: $\langle$ https://doi.org/10.3390/pharmaceuticsi2030265 >. Acesso em: I2 de Julho, 2021.

CARTER, Daniel; LIU, Xueying; TOCKARY, Theofilus; DIRISALA, Anjaneyulu; TOH, Kazuko; ANRAKU, Yasutaka; KATAOKA Kazunori. Targeting nanoparticles to the brain by exploitingthe bloo-brain barrier impermeabilityto selectively label the brainendothelium. Proceedings of the National Academy of Sciences, Aug 2020, 117 (32)

1914I-19150; DOI: I0.1073/pnas.2002016117. Disponível em: 〈https://doi.org/10.1073/pnas.2002016117〉. Acesso em 25 de Agosto, 2021.

CARTER, Daniel; LIU, Xueying; TOCKARY, Theofilus; DIRISALA, Anjaneyulu; TOH, Kazuko; ANRAKU, Yasutaka; KATAOKA Kazunori. Targeting nanoparticles to 
Revista Ibero- Americana de Humanidades, Ciências e Educação- REASE

the brain by exploitingthe bloo-brain barrier impermeabilityto selectively label the brainendothelium. Proceedings of the National Academy of Sciences, Aug 2020, 117 (32)

I914I-19150; DOI: $\quad$ I0.1073/pnas.2002016117. Disponível em: 〈https://doi.org/10.1073/pnas.2002016117〉. Acesso em 25 de Agosto, 2021.

CHOI, James; SELERT, Kirsten Selert; VLACHOS, Fotios; WONG, Anna; KONOFAGOU, Elisa. Non-invasive and localized neuronal delivery using short ultrasonic pulses and Microbubbles. Proceedings of the National Academy of Sciences of the United States of America. October 4, $201 \mathrm{I}$ Io8 (40). Disponível

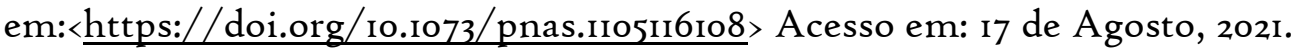

COURTET, Philippe; University Hospital, Montpellier. Exploration of the Relationship Between Inflammation and Integrity of the Blood-Brain Barrier in Suicidal Behaviour (IBIS). ClinicalTrials.gov Identifier: NCTo4137458. (202I) US Department of Health and Human Services, National Institutes of Health. Disponível em: $\langle$ clinicaltrials.gov/ct2/show/NCTo4137458 $>$ Acesso em: 5 de setembro, 2021.

CUMMINGS, Jeffrey; LEE, Garam; RITTER, Aaron; SABBAGH, Marwan; ZHONG, Kate. Alzheimer's disease Drug development pipeline: 16 July 2020. Alzheimer's

Association. Disponível em: < https://doi.org/10.1002/trc2.12050>. Acesso em 25 de Junho, 2021.

DE FALCO, Anna; CUKIERMAN, Daphne; HAUSER-DAVIS, Rachel; REY, Nicolás. Doença de Alzheimer: Hipóteses etiológicas e perspectivas de tratamento. Revista Química Nova. 39 (I). Jan 2016. Disponível em: 〈https://doi.org/10.5935/o100-4042.20150152 > Acesso em 21/o6/2021.

FORNY-GERMANO, Leticia et al.; Alzheimer's disease-like pathology induced by amyloid-beta oligomers in non-humans primates. J Neurosci. The Journal of Neuroscience. 
Revista Ibero- Americana de Humanidades, Ciências e Educação- REASE

open 2 access

2014

Oct

$8 ; 34(4 I)$.

Disponível

em:

〈DOI: https://doi.org/I0.1523/JNEUROSCI.1353-I4.2014> Acesso em: I2 de Abril, 202I.

FORNY-GERMANO, Leticia et al.; Erratum Erratum: Forny-Germano et al., “Alzheimer's Disease-Like Pathology Induced by Amyloid- $\beta$ Oligomers in Nonhuman

Primates". J Neurosci. The Journal of Neuroscience. I4 October 2020, 40 (42). Disponível em:< DOI: 〈https://doi.org/I0.1523/JNEUROSCI.1827-20.2020〉 Acesso em: i2 de Abril, 2021.

GARRIDO-PRADALIE, Emilie; Assistance Publique Hopitaux De Marseille. BloodBrain Barrier Evaluation In Nephrology (BREIN) (BREIN). ClinicalTrials.gov Identifier: NCTo4328415 (2021). US Department of Health and Human Services, National Institutes of Health.

Disponível

em: 〈www.clinicaltrials.gov/ct2/show/NCT04328415〉 Acesso em io de setembro, 2021 GAWDI, Rohin; EMMADY, Prabhu. Physiology, Blood Brain Barrier. In: StatPearls. StatPearls Publishing, Treasure Island (FL); 2020. PMID: 32491653. Disponível em: 〈https://europepmc.org/article/nbk/nbk55772I $\rangle$ Acesso em: 22 de Agosto, 202I.

KATZUNG, Bertram; TREVOR, Anthony. Farmacologia básica e clínica. 13 ed. Porto Alegre: McGraw-Hill, 2017. 1202 p.

KONOFAGOU, Elisa; Columbia University Irving Medical Center/NYPH; National Institute on Aging (NIA). Non-invasive Blood-brain Barrier Opening in Alzheimer's Disease Patients Using Focused Ultrasound. ClinicalTrials.gov identifier: NCTo4I18764 (2020). US Department of Health and Human Services, National Institutes of Health. Disponível em: $<$ clinicaltrials.gov/ct2/show/NCTo4I18764 > Acesso em: 02 de Agosto, 2021.

MENDONÇA, Monique; SOARES, Edilene; DE JESUS, Marcelo; CERAGIOLI, Helder; FERREIRA, Mônica; CATHARINO, Rodrigo; CRUZ-HOFLING; Maria. Reduced graphene oxide induces transient blood-brain barrier opening: an in vivo study. 
Journal of Nanobiotechnology, volume 13, Número do artigo: 78, 2015. Disponível em: $\langle\underline{\text { https://doi.org/I0.II86/sI295I-015-0143-z }\rangle . ~ A c e s s o ~ e m: ~} 03$ de Agosto, 2021.

MICHELLE, Clark et al; Farmacologia ilustrada. $5^{\circ}$ ed. Porto Alegre, RS: Artmed, 2013. 6II p.

MOLINUEVO, Jose; DE LA TORRE, Rafael; Parc de Salut Mar; Fundacion IMIM;

Barcelonabeta Brain Research Center. Prevention of Cognitive Decline in ApoE4 Carriers

With Subjective Cognitive Decline After EGCG and a Multimodal Intervention ((PENSA)). ClinicalTrials.gov identifier: NCT03978052 (2020). US Department of Health and Human Services, National Institutes of Health. Disponível em: $<$ clinicaltrials.gov/ct2/show/NCT03978052 $>$ Acesso em: or de setembro, 2021. 
GAWDI, Rohin; EMMADY, Prabhu. Physiology, Blood Brain Barrier. In:

PARDRIDGE, William. Drug transport across the blood-brain barrier. JCBFM Journal of Cerebral Blood Flow \& Metabolism, 2012 Nov; 32(II): 1959-1972. Doi: 10.1038/jcbfm.2012.126. Disponível em: 〈https://doi.org/10.1038/jcbfm.2012.126〉. Acesso em: 22 de agosto, 2021.

PAULA, Vanessa; GUIMARÃES, Fabiana; DINIZ, Breno; FORLENZA, Orestes. Neurobiological pathways to Alzheimer's disease Amyloid-beta, Tau protein or both?. Views \& Reviews - Dementia \& Neuropsychologia. São Paulo SP. 3 (3) - Jul-Sep 2009. Disponível em: 〈https://doi.org/10.1590/Sig8o$57642009 \mathrm{DN}_{30300003}>$ Acesso em: II de setembro, 2021.

PERONA, Javier; National Research Council, Spain; Hospitales Universitarios Virgen del Rocío. Attenuation of Inflammatory Processes Associated With Alzheimer's Disease After Consumption of Pomace Olive Oil. (ORIVA2). ClinicalTrials.gov Identifier: NCT04559828. (202I) US Department of Health and Human Services, National Institutes of Health. Disponível em: $\langle\underline{\text { clinicaltrials.gov/ct2/show/NCTo4559828 }>}$

RAYMOND Scott; TREAT Lisa, DEWEY Jonathan; MCDANNOLD Nathan; HYNYNEN Kullervo, BACSKAI Brian. Ultrasound enhanced delivery of molecular imaging and therapeutic agents in Alzheimer's disease mouse models. PLoS One. 2008, May I4;3(5):e2175. Disponível em:〈doi: I0.1371/journal.pone.0002175〉 Acesso em: 03 de setembro, 2021.

SHARMA, Kamlesh. Cholinesterase inhibitors as Alzheimer's therapeutics. Journal: Molecular Medicine Reports, June II, 2019. Disponível em: $\langle$ https://doi.org/10.3892/mmr.2019.10374〉. Acesso em: 25 de Agosto, 2021.

SILVERTHORN, Dee Unglaub; Fisiologia humana: uma abordagem integrada. 7. ed. Porto Alegre: Artmed, 2017. 960 p.

VÁZQUEZ-ROSA, Edwin et al.; $\mathrm{P}_{7} \mathrm{C}_{3}-\mathrm{A}_{20}$ treatment one year after $\mathrm{TBI}$ in mice repairs the blood-brain barrier, arrests chronic neurodegeneration, and restores 
cognition. October 2I, 2020, II7 (44). Proceedings of the National Academy of Sciences. Disponível em: 〈https://doi.org/10.1073/pnas.2010430117〉 Acesso em: 3I de Agosto, 2021.

VENTURA, Ana; ABREU, Paula; FREITAS, Rodrigo; SATHLER, Plínio; LOUREIRO, Natália; CASTRO, Helena. Sistema colinérgico: revisitando receptores, regulação e a relação com a doença de Alzheimer, esquizofrenia, epilepsia e tabagismo. Archives of Clinical Psychiatry (São Paulo) 37 (2). 2010 •. Disponível em: 〈https://doi.org/10.1590/Soror-60832010000200007〉. Acesso em 25 de Junho, 2021.

WISHART, David; FEUNANG, Yannick; GUO, An; LO, Elvis; MARCU, Ana; GRANT Jason; SAJED, Tanvir; JOHNSON, Daniel; LI, Carin; SAYEEDA, Zinat; ASSEMPOUR, Nazanin; IYNKKARAN, Ithayavani; LIU, Yifeng; MACIEJEWKI, Adam; GALE, Nicola; WILSON, Alex; CHIN, Lucy; CUMMINGS, Ryan; LE, Diana; PON, Allison; KNOX, Craig; WILSON, Michael. DrugBank 5.0: a major update to the DrugBank database for 2018. Nucleic Acids Res, 2017, 4;46(Di):Dio74-Dio82. Doi: 10.1093/nar/gkxio37. Disponível em: 〈https://doi.org/I0.1093/nar/gkxio37〉 Acesso em: 20 de Agosto, 202I.

WOOD, Jhon; Children's Hospital Los Angeles. Neurovascular Complications and White Matter Damage in Acquired Anemias. ClinicalTrials.gov Identifier: NCTo4320966. US Department of Health and Human Services, National Institutes of Health. Disponível em: < clinicaltrials.gov/ct2/show/NCT04320966> Acesso em: 12 de setembro, 2021. 